\title{
An Overview of Recent KDP Damage Experiments and Implications for NIF Tripler Performance
}

\author{
M. Runkel, R. Jennings, J. DeYoreo, \\ W. Sell, D. Milam, N. Zaitseva, \\ L. Carmen, W. Williams
}

This paper was prepared for submittal to the Third Annual International Conference on Solid State Lasers for Application (SSLA) to Inertial Confinement Fusion (ICF)

Monterey, California June 7-12, 1998

July 14, 1998

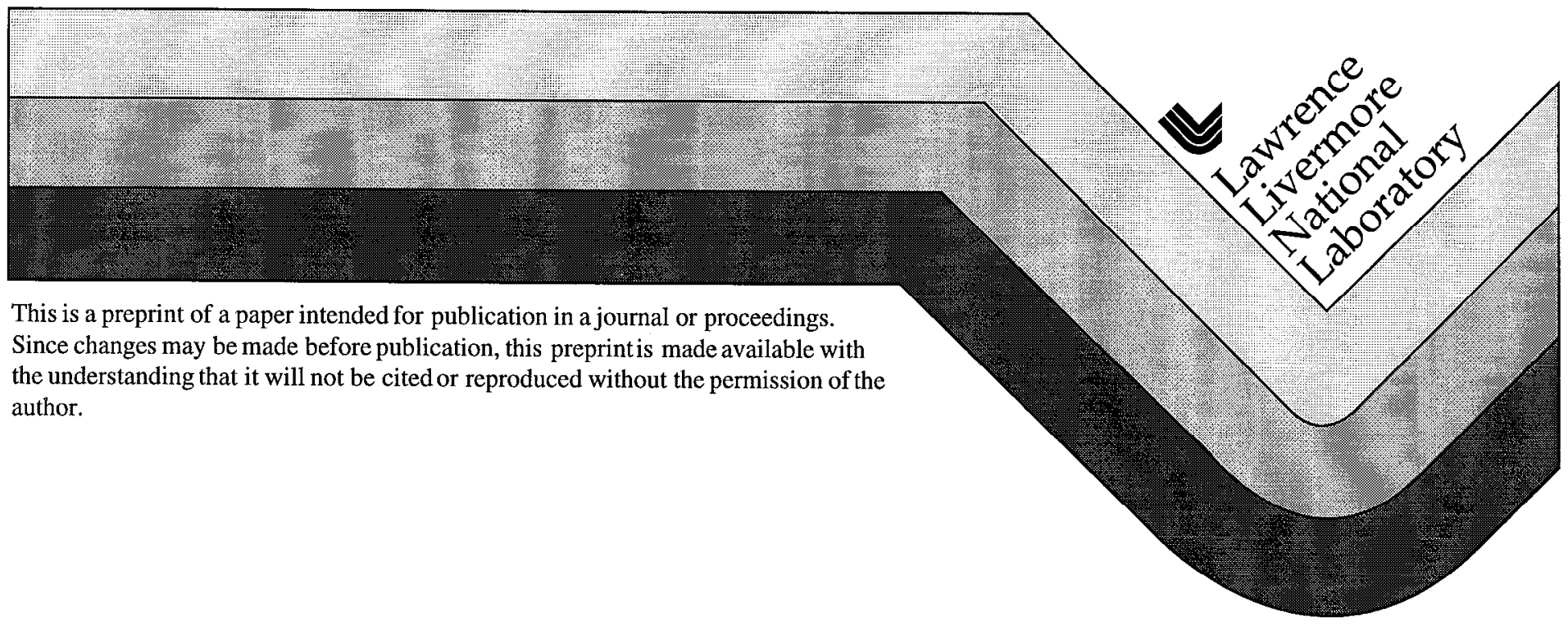




\section{DISCLAIMER}

This document was prepared as an account of work sponsored by an agency of the United States Government. Neither the United States Government nor the University of California nor any of their employees, makes any warranty, express or implied, or assumes any legal liability or responsibility for the accuracy, completeness, or usefulness of any information, apparatus, product, or process disclosed, or represents that its use would not infringe privately owned rights. Reference herein to any specific commercial product, process, or service by trade name, trademark, manufacturer, or otherwise, does not necessarily constitute or imply its endorsement, recommendation, or favoring by the United States Government or the University of California. The views and opinions of authors expressed herein do not necessarily state or reflect those of the United States Government or the University of California, and shall not be used for advertising or product endorsement purposes. 


\title{
An overview of recent KDP damage experiments and implications for NIF tripler performance
}

\author{
M. Runkel, R. Jennings, J. DeYoreo, W. Sell, D. Milam, N.Zaitseva, \\ L. Carmen, W. Williams \\ Lawrence Livermore National Laboratory \\ P.O. Box 808, L-250 \\ Livermore, CA 94550 \\ Phone: (510)-424-2210 \\ FAX: (510)-422-3361
}

\begin{abstract}
Considerable attention has been paid over the years to the problem of growing high purity KDP and KD*P to meet damage threshold requirements of ICF lasers at LLNL. The maximum fluence requirement for $\mathrm{KD} * \mathrm{P}$ triplers on the National Ignition Facility (NIF) is $14.3 \mathrm{~J} / \mathrm{cm}^{2}$ at $351 \mathrm{~nm}$ in a $3 \mathrm{~ns}$ pulse. Currently KD*P (conventional or rapid grown) cannot meet this requirement without laser (pre)conditioning. In this overview, recent experiments to understand laser conditioning and damage phenomena in $\mathrm{KDP}$ and $\mathrm{KD} * \mathrm{P}$ will be discussed. These experiments have lead to a fundamental revision of damage test methods and test result interpretation. In particular, the concept of a damage threshold has given way to measuring performance by damage distributions using millimeter sized beams. Automated R/1 (conditioned) damage tests have shown that the best rapidly grown KDP crystals exhibit the same damage distributions at the best conventionally grown KD*P. Continuous filtration of the growth solution and post growth thermal annealing are shown to increase the damage performance as well.
\end{abstract}

In addition, centimeter size beams from multijoule lasers have been used to study stepwise laser conditioning in KDP. These tests have shown that an increase in the damage threshold of $\sim 1.5 \mathrm{X}$ is attainable with $8-12$ shots of increasing fluence. The experiments show that the damage density (pinpoints $/ \mathrm{mm}^{3}$ ) evolves exponentially and once formed, the micron sized bulk pinpoints remain stable against increases in local fluence.

The information obtained from damage distributions and conditioning studies has been used with model NIF spatial profiles to determine the probability of damage and the local pinpoint density generated in a tripler. Calculations based on test data have shown that for well conditioned, high quality rapid growth KDP or conventional growth $\mathrm{KD} * \mathrm{P}$ the damage probability is less than $3 \%$. Furthermore, the fluence profiles expected on NIF lead to only small numbers of generated pinpoints which are not expected to adversely affect NIF operations.

To check the validity of the results, the $37 \mathrm{~cm} \mathrm{KD*P}$ tripler from the Beamlet laser was mapped for damage. The inspection revealed pinpoint densities of the order of predicted by the damage evolution calculations.

Keywords: KDP, KD*P, Laser damage, bulk damage

\section{INTRODUCTION}

The evolution of increasingly powerful inertial confinement fusion (ICF) laser systems places severe demands on the damage performance of optical system components. In particular, the current maximum $351 \mathrm{~nm}(3 \omega)$ fluence requirement of $14.3 \mathrm{~J} / \mathrm{cm}^{2}$ placed on KD*P triple harmonic generation crystals of the National Ignition Facility (NIF) exceeds the unconditioned damage threshold of even the best material available. Thus KD*P triplers are considered to be the NIF system fuse. The micron sized voids generated within the bulk material act as scatterers and in high enough density are expected to affect frequency conversion efficiency and wavefront distortion. At a minimum, this will result in energy loss and possibly give rise to a number of adverse effects including damaging "hot" spots in the downstream optical components.

In order for NIF to reach its planned energy output, it was mandated from the outset that the damage performance the triplers would improve to the level that the NIF $3 \omega$ THG redline fluence would be met. In the past, the path to 
conditioning ${ }^{1-6}$. With the shift in crystal growth methods from conventional (slow) growth to rapid growth for production of approximately $600,50 \mathrm{~cm}$ class NIF Pockel's cell and harmonic generation crystals, it was not clear what the effects of these technologies on crystal damage performance would be. To accommodate the extensive damage test requirements resulting from the rapid growth crystal development and subsequent quality assurance testing during NIF crystal production, a dedicated KDP damage test facility was required. The ZEUS damage test facility ${ }^{7}$ consists of a commercially available $10 \mathrm{~Hz}$, Q-switched Nd:YAG laser capable of delivering ramped fluences up to $100 \mathrm{~J} / \mathrm{cm}^{2}$ in millimeter sized beams at both $1064 \mathrm{~nm}(1 \omega)$ and $355 \mathrm{~nm}(3 \omega)$. The system has automated site selection and irradiation capabilities and uses a HeNe scatter detection diagnostic to measure the onset of bulk damage at each test site. The system can test hundreds of sites per sample. This makes it possible to determine the characteristic damage distribution of the sample and forces the results to be interpreted in a probabilistic manner, as opposed to the strict damage threshold of the past. Because of the statistical nature of damage in KDP, hundred site tests give a more intrinsically accurate (from a sampling standpoint) means of determining whether growth and post growth process changes have a real effect on the damage distributions. Also, the tests reveal the low fluence nature of the damage distribution, and make it possible to determine the probable damage performance in a NIF-like beam, despite the fact that the material may not have zero failure probability at the NIF $3 \omega$ redline fluence. This will be discussed in Section 2.

In addition to the extensive research effort undertaken to grow crystals with high initial damage performance, numerous experimental campaigns have been performed to determine the effect of post growth treatment on KDP damage performance. Such methods are required because it is desirable to reduce the NIF redline failure probability to the lowest value possible. Post growth processing is expected to reduce fluctuations in the damage performance within a single crystal as. well as for the ensemble of NIF triplers. These include the effect of thermal annealing and laser conditioning. Literature cited above has shown that thermal conditioning is effective in raising the damage threshold at $1 \omega$ but not at $3 \omega$. Laser conditioning experiments have shown that a $\sim 1.5 X$ increase in damage

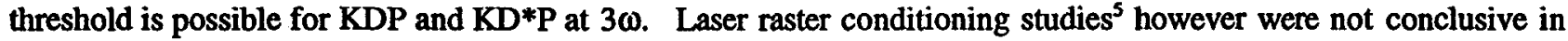
the utility of that technique in raising the damage performance at $3 \omega$. On the other hand, it has long been known that $\mathrm{KDP}$ and $\mathrm{KD} * \mathrm{P}$ can be laser conditioned by ramped fluence sequences, leading to the possibility that the crystals could be conditioned on-line. For this method to be a viable on NIF a shot sequence delivering a maximum level of conditioning for a minimum number of shots was needed. To determine the feasibility and prescription for optimal on-line conditioning a test campaign on the Optical Sciences Laser was undertaken ${ }^{11}$. This laser is a multijoule Nd:Glass facility capable of delivering a centimeter sized $3 \omega$ "top hat" beam to the samples at a rate of 10 shots/day thus mimicking exposure in a NIF-like beam environment. The results of this testing will be discussed in Section 3.

With the extensive amount of experimental data accumulated on ZEUS and OSL and a model of the NIF beam at the triplers, it was possible develop two methods for predicting the probability of failure and amount of damage generated for typical KD*P in a NIF beam. The results of the calculations will be discussed in Section 4 as well as "confirmation" of the predictions based on scatter mapping of a $37 \mathrm{~cm}$ tripler from the Beamlet laser.

\section{ZEUS TEST RESULTS}

\subsection{General features of cumulative failure distributions}

As stated above, the ZEUS damage test results are characteristic of each sample. Information about the distributions can be obtained in two ways. As shown in Figure 1 the test data can be displayed as a cumulative failure distribution (CFD) denoted by the "S" curve. This results from determining the $\sim 100$ individual damage onset fluences for each sample tested and sorting them in ascending order. If the derivative of the CFD is taken (i.e. the distribution is binned), the result is the damage probability distribution. The data shown is for conventionally grown KDP311 which represents the highest damage performance ever measured for KDP or KD*P at LLNL. The CFD curve shows numerous features common to all KDP damage. Specifically, damage tends to be slight at lower fluences and increases in density as fluence goes up. This is indicated by the two photographs accompanying the CFD plot which show scattered HeNe light from damage at low and high fluences. As can be seen in the second photograph, the damage density increases dramatically at high fluences. The second plot shows the damage 
probability distribution. There are two modes to this distribution which can both be fitted fairly well by gaussians at the bin resolution shown. The bimodal distribution indicates that there are two classes of damaging defect for this material with the low fluence mode determining the damage behavior on NIF. Moreover, it is important to note that the low fluence class of defects has not been entirely removed, even for this extraordinary specimen. To date all of the high damage performance crystals whether conventionally or rapidly grown exhibit a similar bimodal distribution.
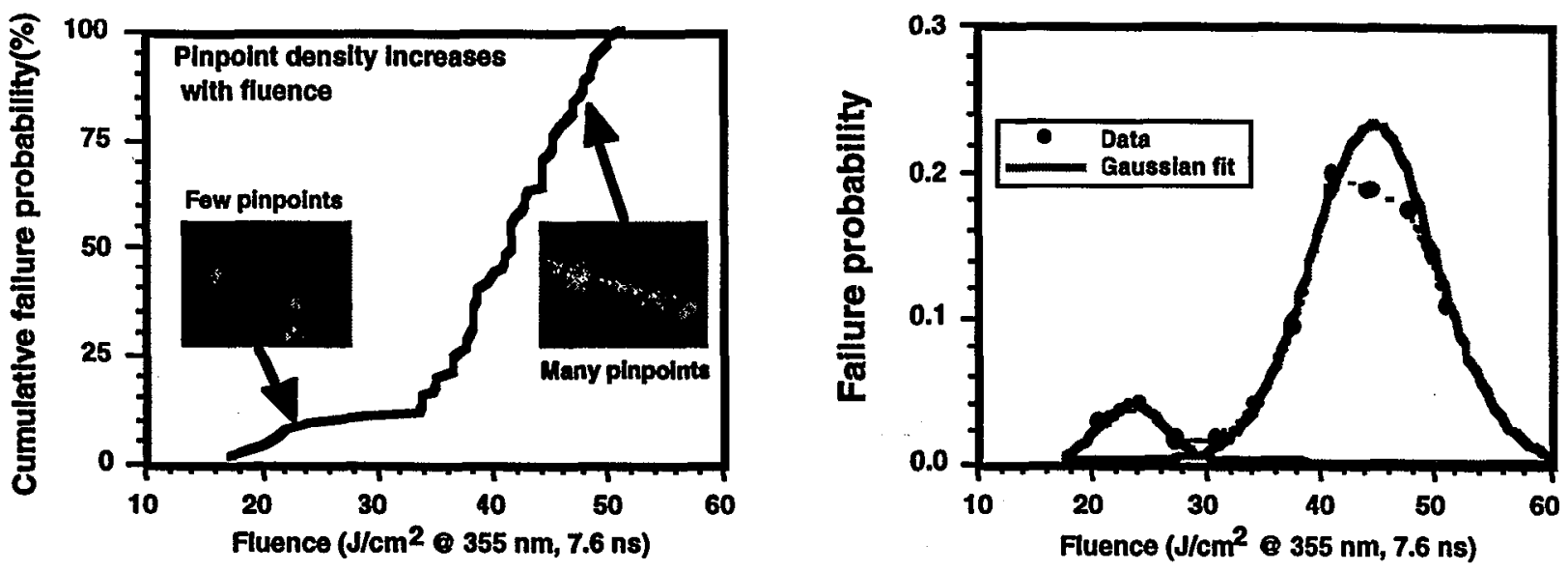

Figure 1. Cumulative failure distribution and associated damage probability for conventionally grown KDP311. This sample represents the best KDP produced for ICF laser applications. The 50\% failure fluence is approximately $44 \mathrm{~J} / \mathrm{cm}^{2}$ at $3 \omega$ in a $7.6 \mathrm{~ns}$ pulse, however there is a long low fluence tail which determines the damage performance on NIF. This feature appears as as the low fluence mode in the damage probability plot on the right.

\subsection{Effects of continuous filtration}

The first implementation of a continuous filtration system for KDP growth was done at LLNL in $1990^{8}$. The study suggested that it was possible to dramatically increase the damage thresholds of crystals grown using this technique. Therefore a continuous filtration system was developed for rapid growth crystals. The system removes growth solution from the tanks, heats it to prevent spontaneous nucleation, filters the solution through 0.02 micron pore filters. The solution is then cooled prior to injection back into the growth tank'. To test the effect of this technology on rapid growth crystals, two samples were grown from ultra high purity starting salts. These salts were obtained by reclaiming the pyramid tops from previously grown KDP boules. It is known that the incorporation of cationic defects is minimized in the pyramidal sector due to the low segregation coefficients which generally results in very high purity material ${ }^{10}$. This material was used to grow crystals under nominally identical growth conditions with and without continuous filtration. Sample KDP210 was grown with prefiltration of the solution by 0.02 micron filters only, while KDP 214 was grown with pre-and continuous filtration of the solution. Figure 2 shows the effect on damage performance. Sample 210 showed a high number of visible bulk scatter sites or inclusions after growth, which were not present in sample 214. For these samples continuous filtration has resulted in an increase in damage performance of approximately $2 X$ over the entire CFD range. Note also that no portion of the CFD for KDP 210 approaches the NIF redline fluence while the CFD for KDP214 indicates that approximately 50\% of the sites would survive NIF redline fluences. As will be seen in the following sections this crystal responded well to thermal annealing and exhibited the best performance of any rapid growth crystal to date. 


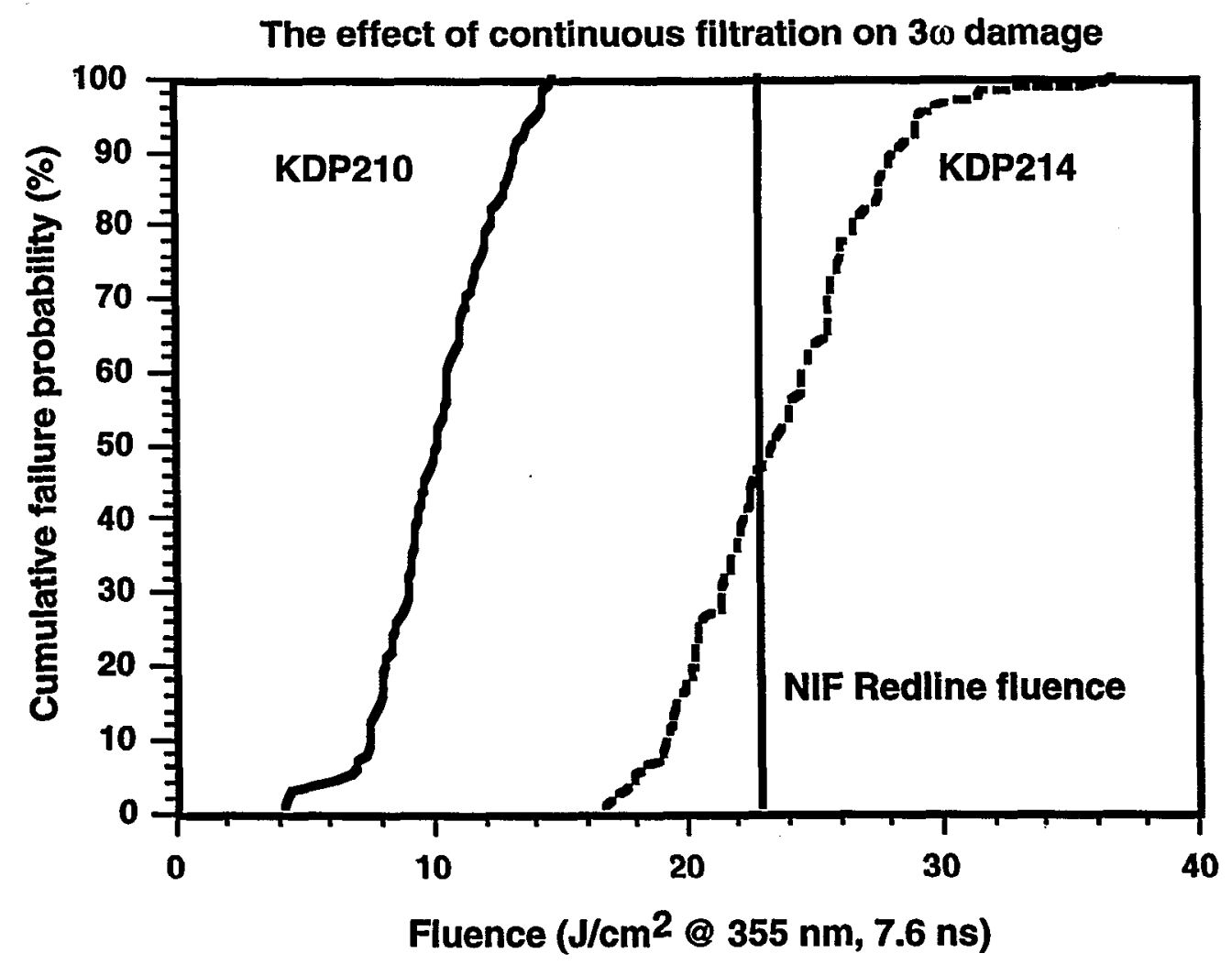

Figure 2. The effect of continuous filtration of the $3 \omega$ damage performance of rapidly grown KDP. The samples were grown under nominally identical conditions with ultra high purity salts. Sample 210 was prefiltered only. which resulted in high numbers of inclusions in the crystal while sample 214 was continuously filtered during growth. Sample 214 represents the highest damage performance of a rapid growth crystal to date.

\subsection{Effects of thermal annealing on damage}

Previous investigations of the effects of thermal annealing on $\mathrm{KDP}$ and $\mathrm{KD} * \mathrm{P}$ damage performance ${ }^{1-4}$ have indicated that it is possible to raise the $1 \omega$ damage threshold to approximately the level the conditioned $(\mathrm{R} / 1)$ damage thresholds by thermal annealing. The typical increase in threshold was on the onder of $1.5 \mathrm{X}$. The $3 \omega$ thresholds, however, were not substantially affected by the annealing process. Once again, the studies were concerned mainly with conventionally grown crystal and the test were done on nonautomated damage facilities with their inherently larger sampling errors. To address the effects of thermal annealing on rapid growth crystals three sets of KDP crystals were annealed at $160 \mathrm{C}$ for various lengths of time up to one month, then damaged tested at both $1 \omega$ and $3 \omega$. In each case, unannealed sister samples were kept as control samples. The samples chosen included KDP60, a relatively early rapid growth boule grown without continuous filtration which contained a fairly high density of visible scatter sites or inclusions. This sample was annealed for one month. In addition, samples from continuously filtered KDP boules 214 was annealed for 5 days. In the case of RG7B the sample was annealed first for 5 days then tested. Based on test results from KDP60 this sample was annealed for an additional 10 days then retested. It should also be pointed out that RG7B represents the first $50 \mathrm{~cm}$ class KDP rapidly grown boule using continuous filtration at LLNL. Results of the tests are shown in Figures 3 and 4 for $1 \omega$ and $3 \omega$ respectively. 


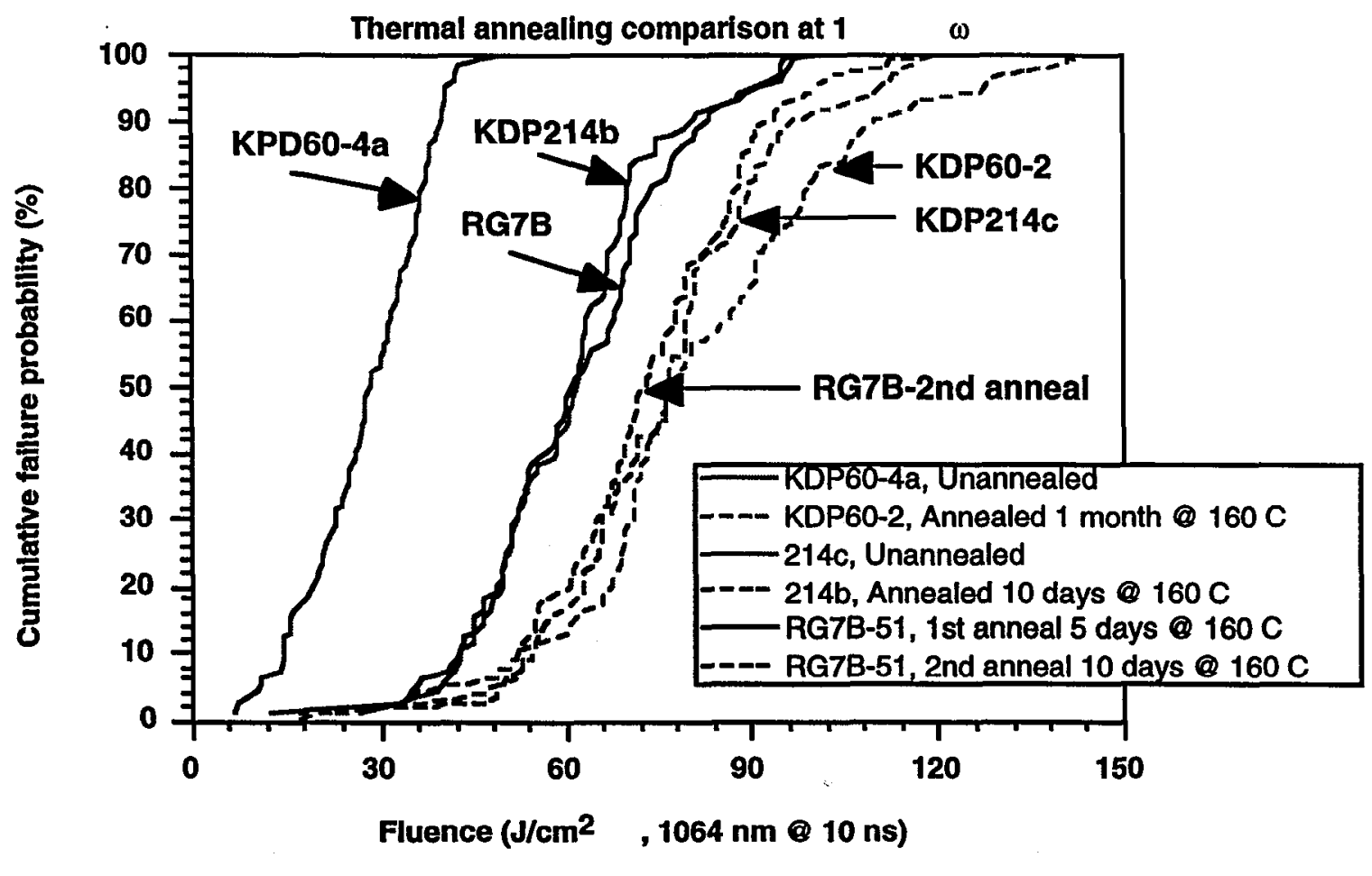

Figure 3. Results of thermal annealing at $1 \omega$. KDP60 showed a $269 \%$ increase in average damage performance while KDP214 and RG7B annealed samples showed respective increases of $18 \%$ and $20 \%$. All the samples were annealed at $160 \mathrm{C}$. KDP60 was annealed for one month.

The results show a dramatic increase in the $1 \omega$ damage performance of KDP60. One month of annealing at $160 \mathrm{C}$ has raised the damage performance approximately $269 \%$ to make this crystal one of the best performing rapidly

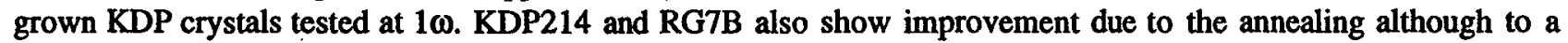
substantially lesser degree. For these samples the increase in 50\% failure probability fluence was $18 \%$ and $20 \%$ respectively. All of the annealed crystals exhibited approximately the same CFD curves. These results indicate that it is possible to achieve damage performance improvement in any rapidly grown KDP crystal at $1 \omega$. At the $1 \omega$ SHG NIF redline fluence ( $32 \mathrm{~J} / \mathrm{cm}^{2}$ at $10 \mathrm{~ns}$ ), all of the annealed samples show failure probabilities on the order of 24\%. Based on these low failure probabilities it is not expected that that damage will be an issue for Pockel's cells and doubler crystals.

For the case of $3 \omega$ damage changes as a result of annealing are not as straightforward. As shown in Figure 4, KDP60 did not exhibit the dramatic change seen at 1 0 . In contrast, KDP214 and RG7B show increases in the 50\% failure probability fluence as a result of annealing, however, the increases are on the order of the fluence measurement precision of ZEUS at $3 \omega( \pm 15 \%)$. This increase is approximately the same magnitude as that seen at $1 \omega$. The CFD curves for KDP214 and RG7B also indicate that the annealing process surpresses the low fluence portion of the curve which results in the low fluence tail seen in all high quality crystals, whether rapidly or conventionally grown. The failure probabilities at the NIF $3 \omega$ redline fluence for these sample are on the order of 26\%. As will be discussed in detail in Section 4 the low failure probabilities will lead only to low levels of benign pinpoint damage on the NIF. Since thermal annealing clearly raises the $R / 1$ damage performance at $1 \omega$ and seems to surpress the low fluence damage probability at $3 \omega$ it is expected that it will be incorporated as a post growth tool for NIF production crystals. 


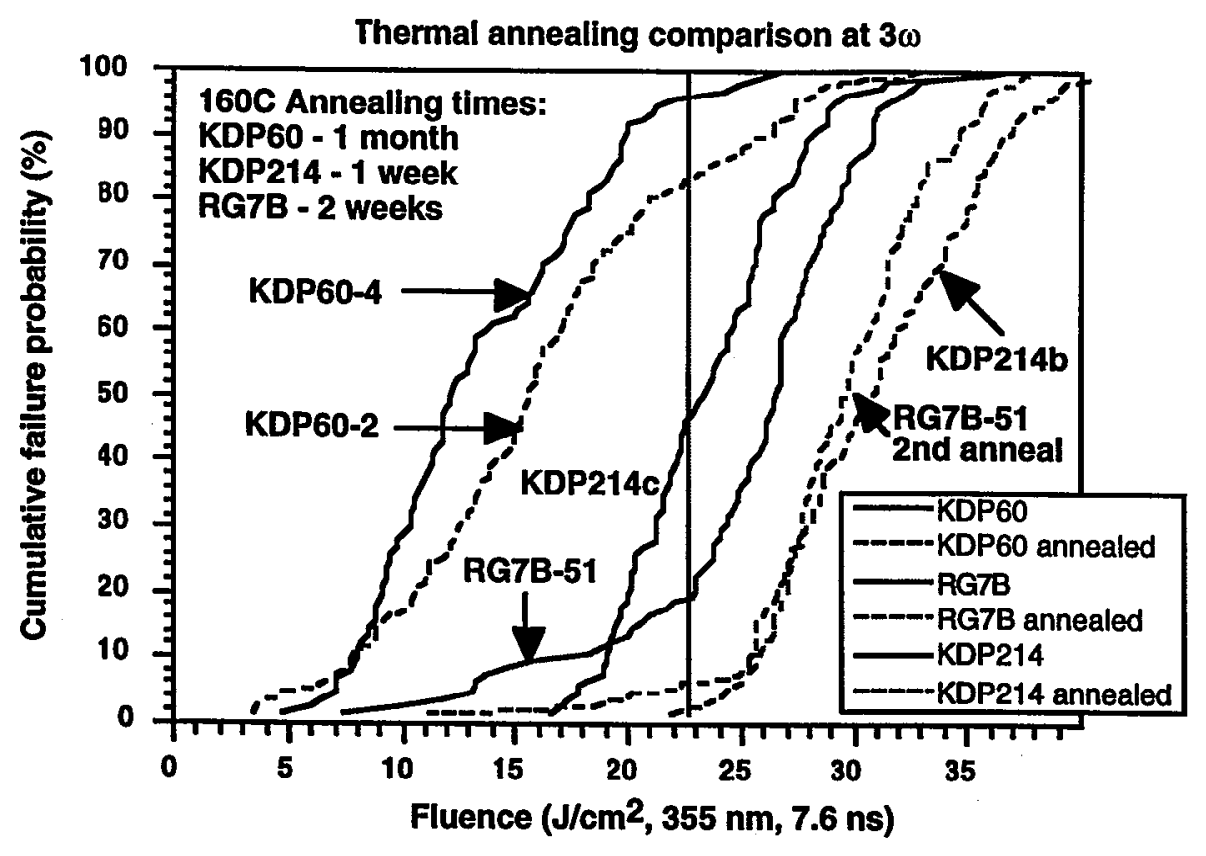

Figure 4. Results of thermal annealing at $3 \omega$. The damage performance of KDP60 has not increased to the same has not responded significantly to the annealing process. In contrast, KDP214 and RG7B show increases in 50\% failure probability on the order of that scen for $1 \omega$. These samples also show supression of the low fluence portion of the CFD. The increase in 50\% failure fluence for KDP214 and RG7B is approximately the same as the ZEUS $3 \omega$ fluence measurement precision of $\pm 15 \%$.

\section{LARGE BEAM ON-LINE CONDITIONING STUDY}

With the exception of a few Beamlet era, conventionally grown KDP boules, the "typical" KDP or KD*P crystal grown today will have significant probability of failure at the $3 \omega$ NIF redline fluence unless treated after growth in some way. Thermal annealing has been shown effective at raising the $1 \omega$ damage threshold thereby allowing virtually any rapidly grown KDP crystal to operate without problem as a Pockel's cell or frequency doubler. For $3 \omega$ damage though, the effects of thermal annealing are less clear cut. It is therefore desirable to pursue other post growth methods of increasing damage performance. The next logical candidate is laser conditioning. The most straightforward method of conditioning a NIF class tripler is via laser raster conditioning where the crystal would be raster scanned at fixed fluence across its clear aperture. A number of full aperture scans at increasing fluences would be required to achieve maximum conditioning. Unfortunately, unpublished LLNL $3 \omega$ raster conditioning studies ${ }^{5}$ on $\mathrm{KDP}$ have not conclusively shown that off-line conditioning by this method is feasible for KD*P. Consequently, investigating the feasibility of on-line conditioning of NIF triplers at $3 \omega$ has become a high priority for the KDP damage group at LLNL.

It should be noted that the automated test techniques used on ZEUS are based on stepwise laser conditioning with a very low fluence ramp $(\sim 0.1 \mathrm{~J} / \mathrm{cm} 2 / \mathrm{s})$ and a high number of shots. These fluence ramps and shot numbers are simply not feasible for ICF class laser systems due to the high cost per shot. On the other hand, if a high degree of laser conditioning could be obtained with a low number of shots and larger fluence increments there would be at least one feasible way of increasing the damage performance of NIF triplers to acceptable levels. A study of on-line conditioning ${ }^{11}$ was undertaken on LLNL's Optical Sciences Laser (OSL) to determine a ramped fluence shot sequence which would yield adequate laser conditioning for NIF triplers. The results of the study on KDP214 are presented here. Essentially, the sample was exposed to various ramped fluence șhot sequences using a $7 \mathrm{~mm}$ diameter top hat beam at $3 \omega$ with a $3 \mathrm{~ns}$ pulse. The shot sequences started at very low fluence and ended at approximately the same average fluence as shown in Figure 5. The single shot/site average fluence was chosen so that substantial pinpoint 
density would be generated. The subsequent shot sequences were chosen to ramp up to this level so that a direct comparison of damage pinpoint density could be made.

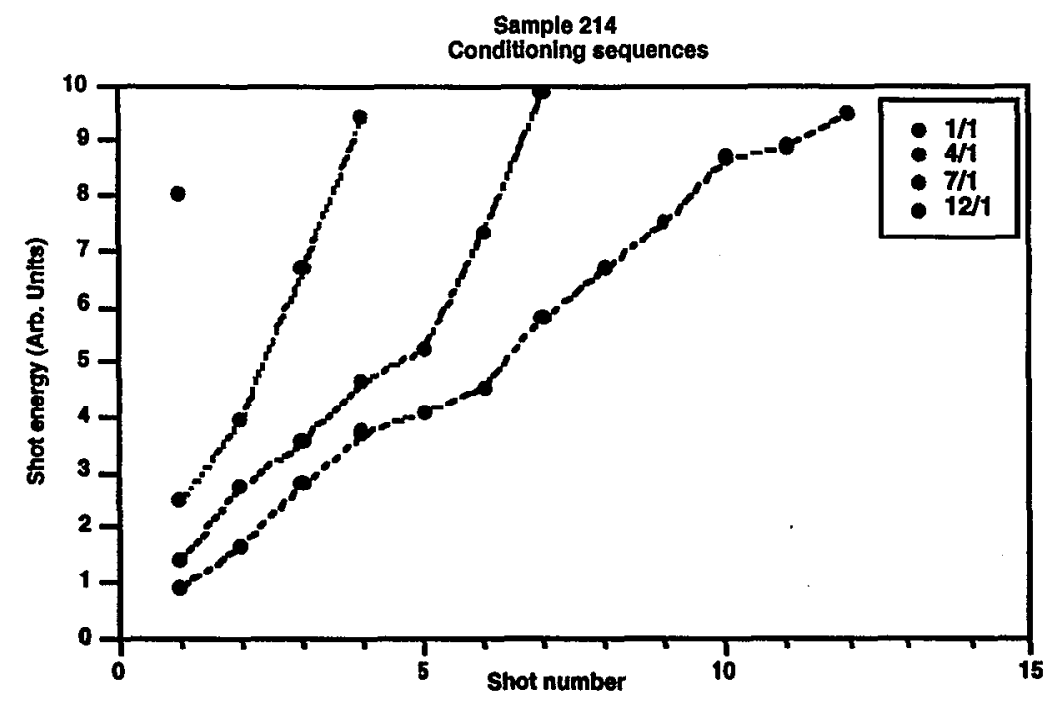

Figure 5. Ramped shot sequences for sample 214. After finding the approximate $1 / 1$ damage threshold, $4 / 1,8 / 1$ and $12 / 1$ ramps to the same approximate average fluence level were attempted. Due to fluctuations in the shot encrgy and the use of fixed attenuators, precise prediction of the average fluence on a given shot was not always possible. As a result the ramped sequences typically received higher average fluence on the final shot than for single shot exposures.

After the exposures, the sample was scatter mapped on the ZEUS system with a 300 micron HeNe beam using 50 micron steps. Figure 6 shows the reduction in pinpoint density as a result of the shot sequences.

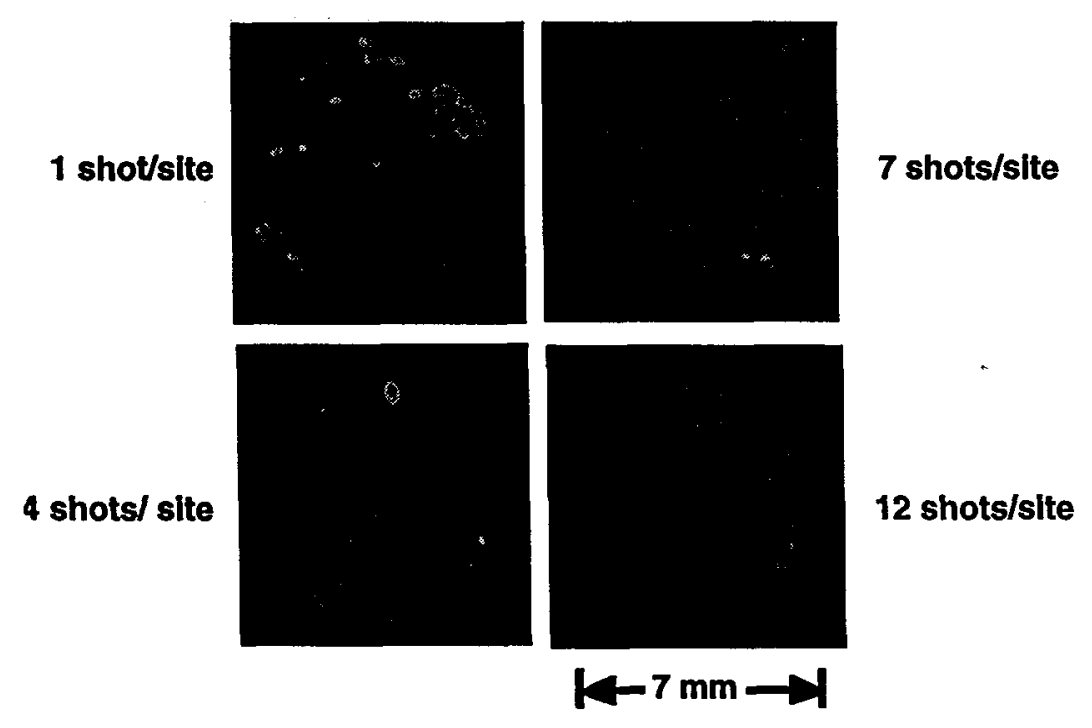

Figure 6. Scatter maps for sample 214. The 1/1 sites shows more severe damage than the ramped sites despite being exposed to approximately $20 \%$ less average fluence. The reduction in damage severity continues to the $12 / 1$ case. 
The pinpoint density obtained from the scatter images was then compared to the local fluence in the spatial profile. Because of the modulation on the beam the damage evolution behavior for each shot sequence could be determined. Figure 7 shows the results of the analysis.

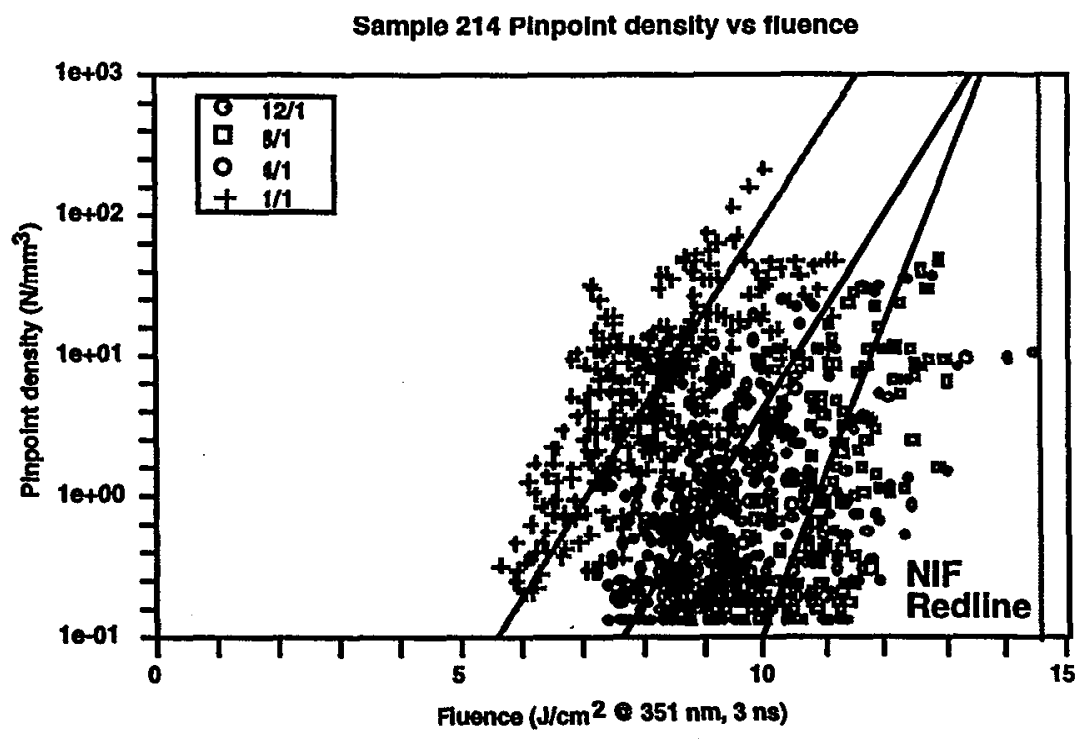

Figure 7. Damage evolution of sample 214 as a result of laser conditioning shot sequences.

The data show clearly the decrease in pinpoint density as a function of shot number. The damage threshold for increasing shot numbers appears to increase up to the $8 / 1$ shot sequence where it saturates. In addition, the pinpoint density follows an exponential evolution where the slope changes between the $4 / 1$ and $8 / 1$ shot sequences. Extrapolated to the NIF redline fluence it appears that there will be in excess of $10^{4}$ pinpoints $/ \mathrm{mm}^{3}$ generated in the bulk of the sample for the $8 / 1$ and $12 / 1$ sequences. The question of whether this pinpoint density will be exceedingly high for NIF operation will be addressed in the Section 4.

These results indicate that full aperture on-line conditioning of NIF triplers should be achievable in approximately 8 shots of increasing fluence in equally spaced intervals. It is safe to conclude that the first KD*P triplers used on the NIF will be adequately conditioned during initial beamline activation. However, considerations of shot cost and scheduling make it clear that on-line conditioning of replacement crystals will not be (very) practical. Therefore there is considerable reason to investigate and develop off-line conditioning technologies such as laser raster conditioning, which seems feasible based on these results, and broadband UV irradiation.

\section{PREDICTING NIF TRIPLER PERFORMANCE}

With test data from the ZEUS and OSL lasers it is possible to predict the damage performance of KDP and KD*P in a NIF-like beam ${ }^{12}$. Once the details of the NIF beam fluence distribution are known there are two approaches which may be taken. First the overlap of the beam fluence distribution and the damage probability distribution may be calculated using tools from reliability analysis ${ }^{13,14}$. Secondly, the damage evolution behavior may be mapped onto the spatial profile to determine the local number of pinpoints generated in the bulk of the material.

Figure 8 shows a PROP92 model spatial profile of the $3 \omega$ beam at the output of the tripler. Ignoring the low fluence tail in the distribution allows the peak to be fitted with a gaussian function with an average of $8.5 \pm 0.81$ $\mathrm{J} / \mathrm{cm}^{2}$. It should be noted that the data above are for the standard full fluence NIF shot and the $12.9 \mathrm{~J} / \mathrm{cm}^{2}$ peak fluence in the beam is $11 \%$ lower than the $14.3 \mathrm{~J} / \mathrm{cm}^{2}$ "redline" fluence for triplers. To examine the damage behavior of the triplers when exposed to a NIF shot with peak fluence at the redline, $11 \%$ was added to each fluence value in the spatial distribution. This resulted in a distribution of $9.05 \pm 1.47 \mathrm{~J} / \mathrm{cm}^{2}$ with a maximum fluence of 14.26 
$\mathrm{J} / \mathrm{cm}^{2}$. The energy content in the beam then became $11.9 \mathrm{KJ}$ for a 192 beam total of $2.3 \mathrm{MJ}$. Fitting the peak of this distribution yielded a mean value (for the fit) of $9.44 \mathrm{~J} / \mathrm{cm}^{2}$ while the standard deviation remains unchanged at $0.81 \mathrm{~J} / \mathrm{cm}^{2}$.

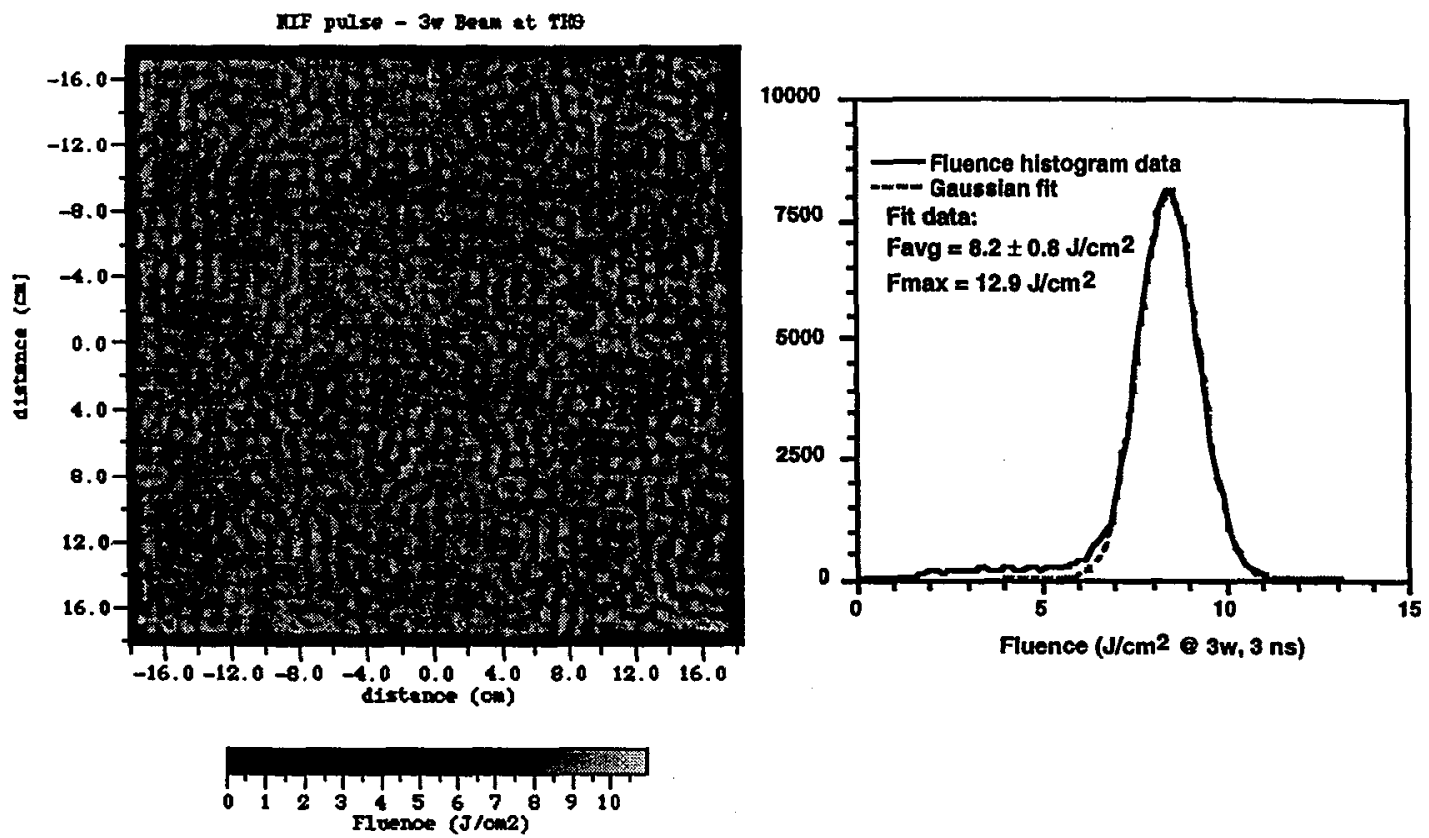

Figure 8. Spatial profile model of NIF beam at output of the $\mathrm{KD} * \mathrm{P}$ tripler and the associated fluence distribution (histogram). The peak can be fitted very well by a gaussian with average of $8.5 \pm 0.81 \mathrm{~J} / \mathrm{cm}^{2}$.

To calculate the overall damage probability for fluence and damage distributions which are gaussian the formula

$$
P(B>D)=\frac{1}{\sqrt{2 \pi}} \int_{\frac{\mu_{z}}{\sigma_{z}}}^{\infty} e^{-t^{2} / 2} d t
$$

can be applied. Here $\mu_{Z}=\mu_{B}-\mu_{D}$ and $\sigma_{Z}^{2}=\sigma_{B}^{2}+\sigma_{D}^{2}$ are the respective mean and standard deviation of the beam(B) and damage (D) distributions. For KDP214 this calculation yields a failure probability of $2.6 \%$ while for the best conventionally grown KDP311 it yields a probability of $0.05 \%$. Because of the large measurement uncertainty in the ZEUS test results these results are essentially indistinguishable.

Predicting the local level of damage in the tripler can be accomplished by mapping the damage evolution behavior onto the individual fluence pixels/cells of the model spatial profile. For KDP214 conditioned with an 7/1 ramp as in the OSL test the best fit damage evolution equation was $\mathrm{N}(\mathrm{F})=5 \times 10^{-10} \exp (2.0 * \mathrm{~F})$. As shown in Figure 9 below, this function leads to low local bulk pinpoint generation. The damage evolution function for unconditioned KDP214 was determined to be $\mathrm{N}(\mathrm{F})=3 \times 10^{-6} \exp \left(1.75^{*} \mathrm{~F}\right)$. A NIF redline shot is predicted to generate a total of 28,000 pinpoints for the unconditioned case but less than 200 for the $7 / 1$ conditioned case.

The reliability analysis calculation indicates that there will be a low overall failure probability for well conditioned NIF triplers while the damage evolution calculations indicate that there will be low local pinpoint generation as well. 


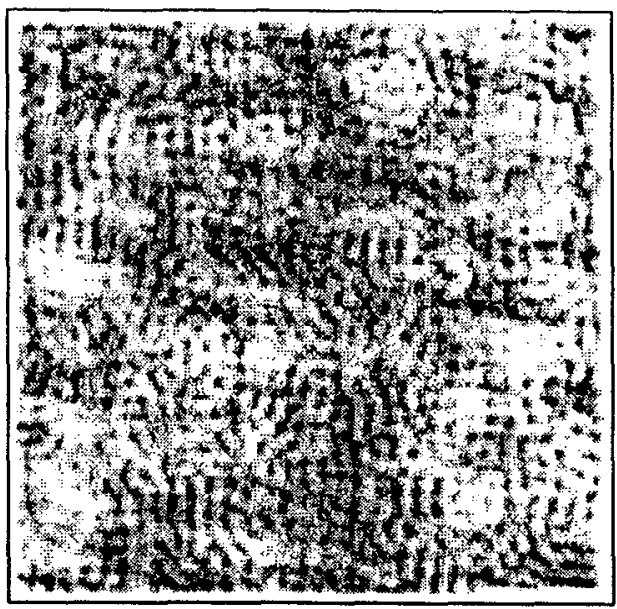

Sample 214 - unconditioned

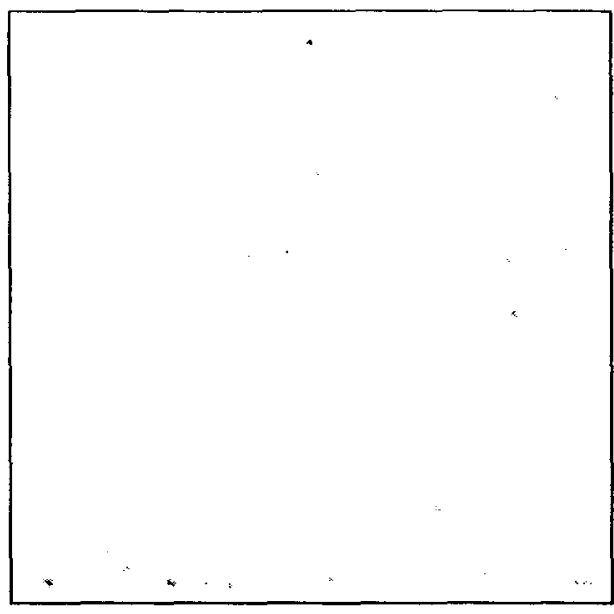

Conditioned

Figure 9. Plots representing the number of pinpoints formed in the bulk of KDP 214 or LL6-56 material for the NIF redline fluence spatial profile. The black areas represent densities which exceed 100 pinpoints in the 0.0095 $\mathrm{cm}^{3}$ cell volume. The color scale is the same for both images.

\section{SUMMARY}

The ZEUS and OSL tests described here have provided definitive information regarding the damage performance of $\mathrm{KDP}$ and $\mathrm{KD} * \mathrm{P}$ on the NIF. In particular the ZEUS test results show the importance of continuous filtration during growth in consistently achieving adequate damage performance. Post growth thermal annealing of KDP crystals at $160 \mathrm{C}$ appears to increase the damage performance of rapidly growth samples by up to $2.7 \mathrm{X}$ at $1 \omega$. The benefits of thermal annealing at $3 \omega$ are not as well defined as the dramatic increases seen at $1 \omega$ were not repeated at $3 \omega$. On the other hand, thermal annealing appeared to surpress the low fluence tail of the CFD at $3 \omega$ thus leading to less expected damage on the NIF. Large area beams from OSL were used to study the feasibility of on-line conditioning for NIF triplers. It was found that substantial conditioning was achievable in 8-12 shots of equal fluence interval. Analysis of scatter density vs. fluence showed that the number of damage sites evolved exponentially. Further, damage sites were stable against increases in fluence.

This data was used in conjunction with model NIF spatial profiles to predict the probability and severity of damage produced by a single shot through well conditioned triplers. It was found that for the best rapidly grown KDP and conventionally grown, Beamlet-era, $\mathrm{KD} * \mathrm{P}$ the damage probability for the entire tripler was less than $3 \%$. In addition, the amount of damage generated in such a tripler was quite low - 165 pinpoints in the entire volume. This is in direct contrast for approximately 28000 pinpoints generated in an unconditioned tripler of the same material. The calculations indicated that well conditioned crystals should survive NIF beam environments with minimal, benign pinpoint damage.

The OSL results indicated the feasibility of on-line conditioning, however, due to cost and time constraints it is unlikely that replacement triplers would be conditioned this way. Consequently, investigation of off-line conditioning techniques is of higher priority. In particular, the OSL test results seem to indicate that laser raster scanning should be effective as a means of conditioning the triplers. Conditioning by broadband pulsed and continuous UV sources will also be pursued.

The data has also provided baseline information for the development of a functional damage specification for NIF operations. Defining this specification will entail a modeling effort which incorporates the damage data generated here. One goal will be to determine the maximum local and cumulative level of damage acceptable for operation of 
the NIF triplers. This work performed under the auspices of the U.S. Department of Energy by Lawrence Livermore National Laboratory under Contract No. W-7405-ENG-48.

\section{REFERENCES}

1. F. Rainer, L.J. Atherton, J.J. DeYoreo, "Laser damage to production- and research grade KDP crystals," in Laser Induced Damage in Optical Materials. SPIE Proceedings, Volume 1848, edited by H.E. Bennet et. al., 46-58, SPIE, Bellingham, WA. 1992

2. F. Rainer et. al., "A historical perspective on fifteen years of laser damage thresholds at LLNL," in Laser Induced Damage in Optical Materials, SPIE Proceedings, Volume 2114, edited by H.E. Bennet et. al., 9-22, SPIE, Bellingham, WA. 1993

3. J. Swain, S. Stokowski, D. Milam, F. Rainer, "Improving the bulk laser damage resistance of potassium dihydrogen phosphate by pulsed laser irradiation," Applied Physics Letters 40(4), pp 350-352, 1982.

4. J. Swain, S. Stokowski, D. Milam, "The effect of baking and pulsed laser irradiation on the bulk laser threshold of potassium dihydrogen phosphate crystals," Applied Physics Letters 41(1), pp 12-14, 1982.

5. F. Rainer, M. Staggs, C. Barker, F. DeMarco, M. Kozlowski, LDG 93-004 (LLNL Internal Memo): "Status of large-area raster conditioning of KDP," 1/25/1993.

6. Y. Sun, C. Li, H. Gong, "Laser treatment to improve the ability to resist laser damage in potassium dihydrogen phosphate crystals," in Laser Induced Damage in Optical Materials, SPIE Proceedings, Volume 1848, pp 574-577, 1992.

7. M. Runkel, M. Yan, J. DeYoreo, N. Zaitseva, "The effect of impurities and stress on the damage distributions of rapidly grown KDP crystals," in Laser Induced Damage in Optical Materials. SPIE Proceedings, 1997

8. K.E. Montgomery, F.P. Milanovich, "High laser damage threshold potassium dihydrogen phosphate crystals," Journal of Applicd Physics, 68(8), 3979-3982, 1990.

9. N. Zaitseva, J. Atherton, R. Rozsa, L. Carmen, I. Smolsky, M. Runkel, R. Ryon, L. James, "The conncction between continuous filtration and dislocation structure of KDP crystals," submitted to Journal of Crystal Growth (?), June 1998

10. M. Yan, R. Torres, M. Runkel, B. Woods, I Hutcheon, N. Zaitseva, J. DeYoreo, "Impurity and laser induced damage in the growth sectors of rapidly grown KDP crystals," SPIE Proceedings, Volume 2966, pp 11-16, 1996

11. M. Runkel, J. DeYoreo, W. Sell, D. Milam, "Laser conditioning study of KDP on the Optical Sciences Laser using large area beams," SPIE Proceedings, 3244, pp 51-63, 1997

12. M. Runkel, "Predicting NIF tripler damage from ZEUS and OSL test results and NIF spatial profiles," LLNL internal memo, NIF-0003944, December 1997

13. E.B. Haugen, Probabilistic Mechanical Design, John Wiley and Sons, New York, 1980

14. S. Zacks, Introduction to Reliability Analysis, Springer Verlag, New York, 1992 